\title{
In Situ Follicular Neoplasia
}

National Cancer Institute

\section{Source}

National Cancer Institute. In Situ Follicular Neoplasia. NCI Thesaurus. Code C138181.

A follicular neoplasia confined to the germinal centers of the lymph nodes, without evidence of disseminated disease. It is characterized by the replacement of a germinal center by neoplastic centrocytes with uniformly intense positivity for BCL2. The surrounding mantle cuff and lymphoid architecture are intact. It has a low rate of progression to follicular lymphoma, but is often associated with prior or synchronous overt lymphoma. 